宋宇, 孟博, 陈学先, 陈号天, 韩梦迪, 程晓亮, 张海霞*

北京大学微米/纳米加工技术国家重点实验室, 北京 100871

*联系人, E-mail: zhang-alice@pku.edu.cn

2015-09-21 收稿, 2015-10-30 修回, 2015-11-13 接受, 2016-01-11 网络版发表

国家自然科学基金(61176103，91323304)、国家高技术研究发展计划(2013AA041102)、北京市自然科学基金(4141002)和北京市科委 (Z141100003814003)资助

摘要柔性固态超级电容器作为一种新型能量存储器件, 与传统平行板电容器相比可以达到更高的能量密度, 相比普通电池则具有更大的功率密度和循环使用寿命, 展示出良好的电化学性能, 并具有高功率密度和循环稳定 性好等优点, 因而受到越来越多的关注, 在可穿戴设备、柔性电子器件等诸多领域有着广泛的应用。目前, 柔性电 极材料的选取与制备是柔性超级电容器研究中十分活跃的方向, 其中, 碳基电极因为具有良好的电导性能、循环 稳定性、高功率密度等特点, 被国内外学术界广泛认可. 本文提出了一种高效、简便制备碳基电极的方法, 得到多 孔富氮纳米片结构的碳电极, 并对不同实验条件下的碳化电极样品做了全面的表征分析与性能测试, 得到了较为 理想的柔性碳化电极样品, 其质量比电容达 $26 \mathrm{~F} / \mathrm{g}$, 面积比电容达 $226 \mathrm{mF} / \mathrm{cm}^{2}$, 等效串联阻抗仅为 $4 \Omega$, 具有很好 的电化学性能.

关键词柔性电极, 超级电容器, 碳化, 电化学比容量

随着社会经济的飞速发展和人民生活水平的不 断提高, 人类对能源的需求也与日俱增. 就目前而 言, 煤炭、石油、天然气等化石能源仍然是主要的能 量来源. 但是, 一方面, 这些传统的化石能源是不可 再生的 ${ }^{[1-3]}$, 最终也将衰竭殆尽; 而另一方面, 化石 燃料燃烧会排放 $\mathrm{CO}_{2}$ 等对环境有害的气体, 导致全球 的生态环境日益恶化, 是社会各界十分关心的问 题 ${ }^{[4]}$. 基于此, 发展高效稳定、环境友好的能量存储 设备对发挥可再生能源优势起着至关重要的作用.

超级电容器, 也称电化学电容器, 是近年来出现 的一种重要的能量存储设备 ${ }^{[5]}$, 它介于传统电容器 与锂离子电池之间, 在结合两者优势方面起着关键 作用, 三者功率密度、能量密度的比较如图1所示. 相

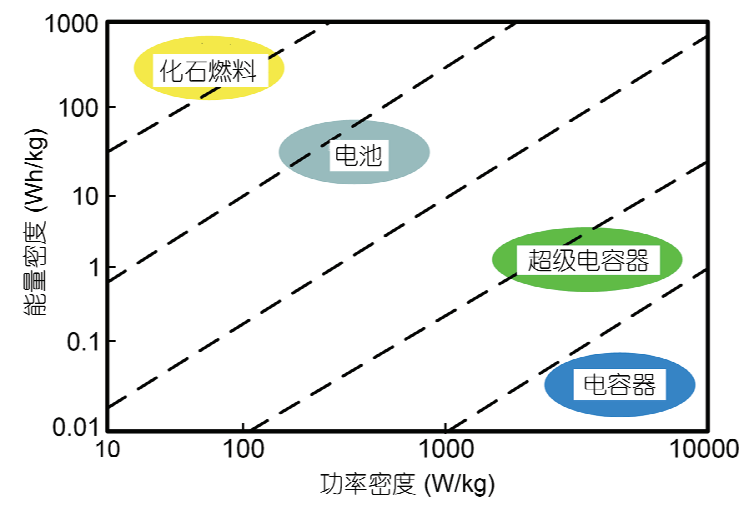

图 1 (网络版彩色)传统电容器、电池与超级电容器能量密度和功率 密度对比图

Figure 1 (Color online) Ragone plot for different energy storage technologies about capacitor, battery and supercapacitor 
比较传统电容器, 超级电容器的比能量密度要高出 几个数量级, 并具有更高的电容值; 而与电池相比, 超级电容器独特的电荷存储机理使其可以在很短的 时间内存储或传输大量的电荷, 因而可以产生更高 的功率密度, 具有充电时间短、充电效率高、循环使 用寿命长以及基本无需维护等特点 ${ }^{[9]}$.

与传统的超级电容器相比, 新兴的柔性超级电 容器同样展示出良好的电化学性能, 并具有高功率 密度和循环稳定性好等优点 ${ }^{[10 ~ 13]}$, 力学上的柔性也 扩展了其应用范围, 在诸如可穿戴设备、生物植人系 统等很多领域有潜在的应用 ${ }^{[14,15]}$. 柔性超级电容器 最主要的部分在于柔性电极材料的选取, 研究者在 制备柔性电极方面付出了很多努力, 也取得了不错 的进展 ${ }^{[16,17]}$. 而如今, 制备具有坚固机械性能和良好 电化学性能的柔性电极仍面临很多挑战, 金属类电 极易被水系电解质侵蚀并且柔性一般 ${ }^{[18]}$, 因此研究 的重点在于非金属电极材料的设计与制备. 其中, 碳 纳米材料的快速发展与优异的机械性能使其在制备 柔性电极方面有很多发展潜力, 从零维的碳纳米颗 粒、一维的碳纳米管到二维石墨烯, 各种类别的碳材 料都具有制备电极的潜在优势 ${ }^{[16,19 ~ 22] . ~}$

由于多数碳电极都受到比表面积、孔径分布等因 素的限制, 因此需要研究新型碳基材料以获得更好 的性能, 如分级多孔结构、二维结构、高比表面积、 杂原子修饰以及高的电导率等. 考虑到可行性及易 获取性等因素, 有必要进一步探索高效简便的合成 新型碳基能量存储设备的方法. 因此, 关于碳基材料 的研究主要基于以下几方面: 具有高比表面积活性 位点以促进充电容量 ${ }^{[23,24]}$; 设计新型纳米结构以缩 短扩散途径, 在电极电解质表面获得最小的物质传 输扩散电阻 ${ }^{[25]}$; 分级多孔结构以产生更快的离子传 输速率 ${ }^{[26,27]}$; 引人缺陷、杂原子或官能团可以增加活 性位点, 可以有效地调控电化学特性 ${ }^{[28]}$. 毫无疑问, 上述特性的多重协同效应可以增强碳基材料在能量 存储应用中的性能. 然而, 通过现有方法同时满足上 述各特性仍是一个巨大难题. 此外, 能简单合成的生 物可再生碳资源的选取也是需要考虑的方向 ${ }^{[29,30]}$.

基于此，考虑到同时满足上述多重协同效应的 可行性以及制备工艺的高效性，本文直接对蚕茧片 进行碳化处理, 无需黏结剂, 制备工艺简单, 得到具 有柔性多孔结构的碳基电极. 该电极为二维纳米片 结构, 其中, 多孔结构为离子提供活性位点, 并作为
离子快速交换的通道，而富氮和多杂原子掺杂和空 位的特性可以提高电化学活性和电导率. 通过高效 简便方法制得新型碳基电极, 进一步增强器件的性 能, 为制备性能良好的柔性超级电容器打下坚实基 础, 满足柔性自供能系统的需求.

\section{1 实验}

(i) 材料. 为满足柔性电极的需要, 直接将整 片虫茧清洗处理后进行碳化，虫茧样品如图2所示. 为了更好地分析不同情况下碳基电极性能的差异, 对不同实验条件制备得到的多孔富氮碳基电极进 行了全面的分析与表征, 比较了各批次实验的性能 差异.

碳化在高温无氧情况下进行，先以碳化时间为 变量, 分析观察碳化时间对电极表征性能的影响, 分 为 $\mathrm{A}, \mathrm{B} 2$ 个批次，进行不同碳化时间下的碳化处理. 样品分别在去离子水与丙酮中润洗 $10 \mathrm{~min}$, 去除表面 的大颗粒杂质与有机杂质, 接下来放人乙醇溶液中, 超声清洗 $15 \mathrm{~min}$, 烘干后为进一步实验做准备. 碳化 工艺使用的是管式炉设备, 管径为 $8 \mathrm{~cm}$, 在 $\mathrm{Ar}$ 气氛 中, 以 $2^{\circ} \mathrm{C} / \mathrm{min}$ 的升温速度, 缓慢升温至 $900^{\circ} \mathrm{C}$, 其中, $\mathrm{A}$ 批次碳化时间为 $1 \mathrm{~h}, \mathrm{~B}$ 批次碳化时间为 $2 \mathrm{~h}$, 分别进 行碳化实验, 并自然冷却至室温. 所得样品用去离子 水冲洗表面后, 放人烘箱中, 在 $120^{\circ} \mathrm{C}$ 下烘干 $12 \mathrm{~h}$, 以 达到最大化去除水分子的目的. B批次柔性固态电极 如图3所示.

为了分析应力对样品硬度的影响，在碳化时间 为 $2 \mathrm{~h}$ 条件下制备另一组碳化样品作为 $\mathrm{C}$ 批次, 经过 清洗烘干后, 将虫茧片以弯曲状态放人直径 $1 \mathrm{~cm}$ 的 石英管中，再放人管式炉中进行与上述B批次相同的 碳化操作, 碳化时间为 $2 \mathrm{~h}$, 得到受应力状态下的碳 化样品，三者制备工艺及制备结果如表1所示.

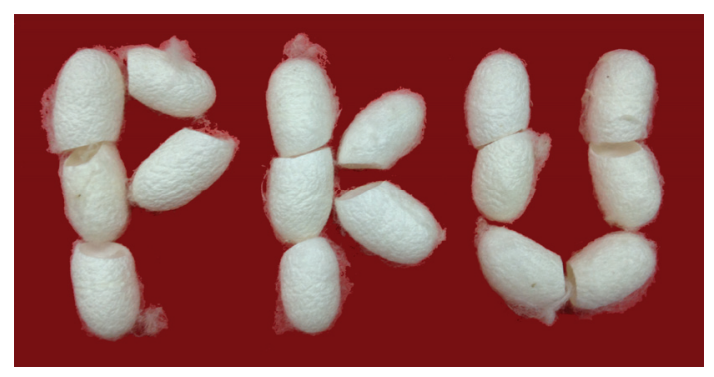

图 2 (网络版彩色) 富含丝素蛋白的蟇茧片样品

Figure 2 (Color online) Natural cocoons rich in silk fibroin fibers 


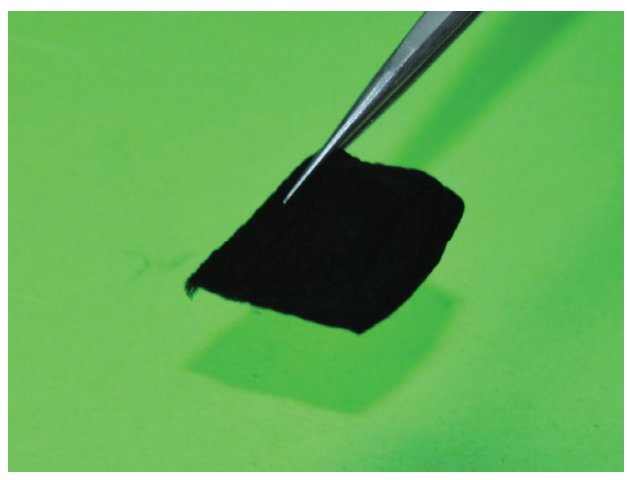

图 3 (网络版彩色) B批次柔性固态碳电极样品

Figure 3 (Color online) Flexible solid-state carbon electrode of sample B

表 1 三批次制备工艺及制备结果

Table 1 Fabrication process and results of different samples

\begin{tabular}{ccccc}
\hline 批次 & 时间 $(\mathrm{h})$ & 管径 $(\mathrm{cm})$ & 柔性度 & 光泽 \\
\hline $\mathrm{A}$ & 1 & 8 & 良好 & 较黑 \\
B & 2 & 8 & 良好 & 黑 \\
C & 2 & 1 & 较差 & 黑 \\
\hline
\end{tabular}

(ii) 性能表征. 对于碳化样品的一系列表征, 使用日本Hitachi公司的S-4800型冷场发射扫描电子 显微镜(SEM)进行SEM电子显微镜实验, 观察表征碳 化后电极的表面形貌; X射线光电子能谱分析(XPS) 在日本Kratos公司Axis Ultra多功能能谱仪上进行, 对表面几纳米范围内的各种元素进行测量和分析; $\mathrm{X}$ 射线衍射 (XRD) 性能测试, 使用美国 FEI 公司的 Quanta 600型X射线衍射仪, 衍射角范围 $20^{\circ} \sim 60^{\circ}$, 主 要对碳元素的晶面指数进行分析与讨论.

制得的多批次柔性碳基电极的电化学性能表征, 在上海辰华CHI660E型电化学工作站上进行, 主要 测试方法是循环伏安法和交流阻抗谱法. 以 $1 \mathrm{~mol} / \mathrm{L}$ 的 $\mathrm{H}_{2} \mathrm{SO}_{4}$ 酸性溶液作为电解液, $\mathrm{Ag} / \mathrm{AgCl}$ 电极 (内含饱 和 $\mathrm{KCl}$ 溶液) 作为参比电极, 对电极使用铂丝电极, 建 立三电极测试体系, 测试条件为室温.

\section{2 结果与讨论}

\section{1 多孔富氮碳电极的结构分析}

为了比较不同碳化时间、碳化作用下元素分布以 及各元素成键的种类与比例, 首先进行了XPS测试, 并对 C $1 \mathrm{~s}$ 轨道能谱和 $\mathrm{N} 1 \mathrm{~s}$ 轨道能谱做了充分的论证 与分析, 相关能谱如图4所示. 从图4(a)和(b)中可以看到明显的 C, O, N 3 种元 素的能谱峰, 说明这3种元素大量存在于样品中, 但 是图 4(a)中 $O$ 元素比例很高, 而 $\mathrm{O}$ 元素比例过高会削 弱电极性能, 对电阻值、电容能力带来负面影响, 并 且在 $N$ 能谱峰的左侧有较为明显的杂质峰, 说明碳化 过程不完全. 而图4(b)中 $\mathrm{O}$ 元素能谱峰明显下降, 杂 质峰有所衰减, 侧面反映出碳化程度改善, 电极性能 有所好转. 为了验证元素比例的变化, 进行了相关数 据处理, 其中 $\mathrm{A}$ 批次碳化样品中 $\mathrm{C}, \mathrm{N}, \mathrm{O}$ 的质量分数 分别为 $82.26 \%, 1.59 \%$ 和 $10.82 \%$, 而 $\mathrm{B}$ 批次碳化样品 中C, N, O的质量分数则为 $89.06 \%, 2.32 \%$ 和 $5.87 \%, \mathrm{C}$ 元素比例明显增加, $\mathrm{O}$ 元素比例大幅下降, 证实了碳 化程度的差异.

在此能谱图的基础上, 进一步分析 $\mathrm{N} 1 \mathrm{~s}$ 轨道能 谱. $\mathrm{N} 1 \mathrm{~s}$ 能谱可以由 2 个能谱峰值拟合, 分别是位于 $398.2 \mathrm{eV}$ 的吡啶型 $\mathrm{N}(\mathrm{N}-6$ 官能团)和位于 $400.9 \mathrm{eV}$ 的四 价型 $\mathrm{N}$ (N-Q官能团), 说明碳化过程下虫茧中的 $\mathrm{N}$ 原 子会与 $\mathrm{C}$ 原子结合, 转化成不同的 $\mathrm{C}-\mathrm{N}$ 官能团, 主要 包含上述 2 类. 其中, 吡啶型 $\mathrm{N}$ 为 $\mathrm{N}$ 原子与石墨烯层边 缘的 $\mathrm{C}$ 原子结合, 而四价型 $\mathrm{N}$ 则代表 $\mathrm{N}$ 原子与石墨烯 层内部的 $\mathrm{C}$ 原子结合. 研究结果表明, 与位于石墨烯 层内部的 $\mathrm{C}$ 原子结合的 $\mathrm{N}$ 原子 $(\mathrm{N}-\mathrm{Q}$ 官能团) 可以增强 碳材料的电化学性能, 而与石墨烯边缘 $\mathrm{C}$ 原子结合的 $\mathrm{N}$ 原子(N-6官能团)则不会增强, 所以 $\mathrm{N}-\mathrm{Q}$ 官能团比例 越高, 对于促进电极能力的帮助越大. XPS能谱的分 析结果可以看出, 对于 A批次碳化样品, N-Q官能团 比例为 $86.63 \%, \mathrm{~N}-6$ 官能团比例则为 $13.37 \%$; 而对于 $\mathrm{B}$ 批次碳化样品, N-Q官能团比例上升为 $88.88 \%, \mathrm{~N}-6$ 官能团比例减小为 $11.12 \%$, 说明 $2 \mathrm{~h}$ 下 $\mathrm{N}-\mathrm{Q}$ 官能团比 例更高, 碳化程度更完全, 电极的电化学性能进一步 提升. 同时, $\mathrm{N}$ 原子的存在可以增强离子的传输能力, 提供活性位点, 带来的空穴可以增加吸附电荷的能 力, 提升电化学性能. 此外, 对 C $1 \mathrm{~s}$ 轨道能谱做了同 样的拟合分析, 2 组样品 $\mathrm{C}$ 原子成键类型都是 $\mathrm{C}-\mathrm{C}$, $\mathrm{C}-\mathrm{N}, \mathrm{C}-\mathrm{O}$ 和 $\mathrm{C}=\mathrm{O}$ 键, 拟合度很好, 显示了 $\mathrm{C}$ 原子的存 在形式与结构关系, 说明了碳化的可行性与准确性.

由于XPS测试的只是表面几纳米内元素分布的 状态, 为了更为全面地分析 2 批次样品元素组成及比 例的差异, 对其进行了元素分析测试, 得到了 $\mathrm{C}, \mathrm{N}$, $\mathrm{H}$ 各元素的比例, 并将所得结果与XPS测试结果进行 了对比(表2). 结果显示, 同一批次样品, 2 种测试下 各元素比例基本一致，而对于同一种测试，A批次碳 

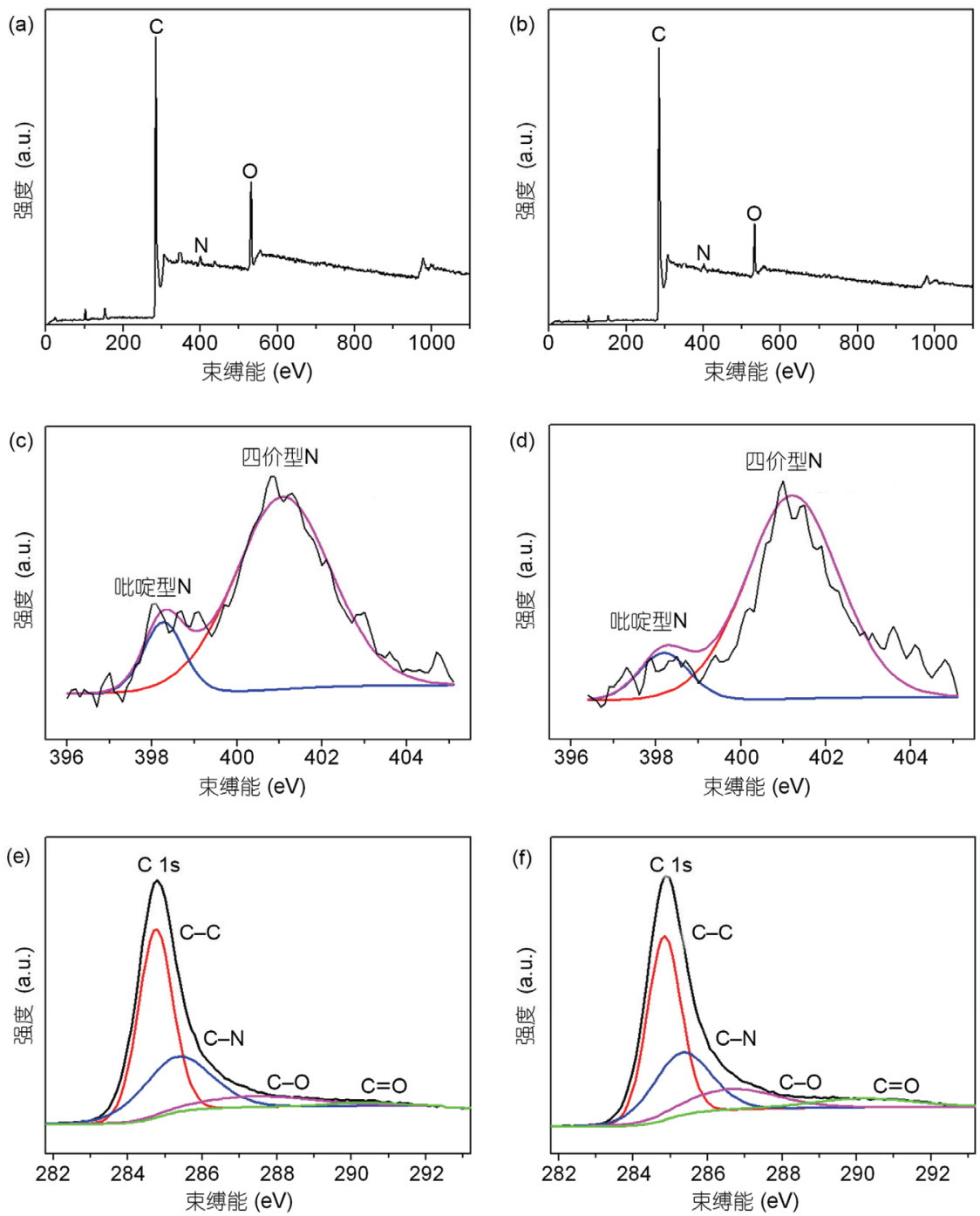

图 4 (网络版彩色)(a), (b) 样品整体能谱峰分布图; (c), (d) N 1s 轨道能谱峰分布图; (e), (f) C 1s轨道能谱峰分布图. (a), (c), (e) A批次样品; (b), (d), (f) B批次样品

Figure 4 (Color online) (a), (b) The whole XPS survey spectrum; (c), (d) high-resolution spectrum of N 1s XPS peak; (e), (f) high-resolution spectrum of C 1s XPS peak. (a), (c), (e) Samples A; (b), (d), (f) samples B

表 2 不同批次下不同测试方法的各元素比例

Table 2 Different samples elemental analysis results under different test methods

\begin{tabular}{cccccc}
\hline \multicolumn{2}{c}{$\begin{array}{c}\text { 分析结果 } \\
\text { (质量百分比) }\end{array}$} & $\mathrm{C}$ & $\mathrm{N}$ & $\mathrm{O}$ & $\mathrm{H}$ \\
\hline \multirow{2}{*}{ 元素分析 } & $\mathrm{A}$ & 84.97 & 1.96 & - & 1.27 \\
& $\mathrm{~B}$ & 85.65 & 2.13 & - & 1.26 \\
\multirow{2}{*}{ XPS } & $\mathrm{A}$ & 82.26 & 1.59 & 10.82 & - \\
& $\mathrm{B}$ & 89.06 & 2.32 & 5.87 & - \\
\hline
\end{tabular}

化样品在 $\mathrm{C}, \mathrm{N}$ 元素的含量上都低于B批次碳化样品, $\mathrm{H}$ 元素在碳化过程中变化不大, 这也与之前的分析相
吻合，进一步阐释了碳化对于样品元素组成的影响, 即碳化时间越长, $\mathrm{C}, \mathrm{N}$ 元素比例越高.

此外, $2 \mathrm{~h}$ 碳化样品的XRD相位测试结果如图5所 示, $20^{\circ} \sim 60^{\circ}$ 之间有 2 个明显的衍射峰 $24^{\circ}$ 和 $44^{\circ}$. 用 JCPDS 41-1426标准卡片比较后发现, 2 个峰值分别对 应 $\mathrm{C}(002)$ 晶面和 $\mathrm{C}(101)$ 晶面, 都属于六角形碳晶面, 说明经过碳化后 $\mathrm{C}$ 原子有了一定程度的晶化, 具有定 向态, 分布较为规则.

\section{2 多孔富氮碳电极的形貌表征}

为了对碳化电极形貌进行观察与表征, 对碳化 


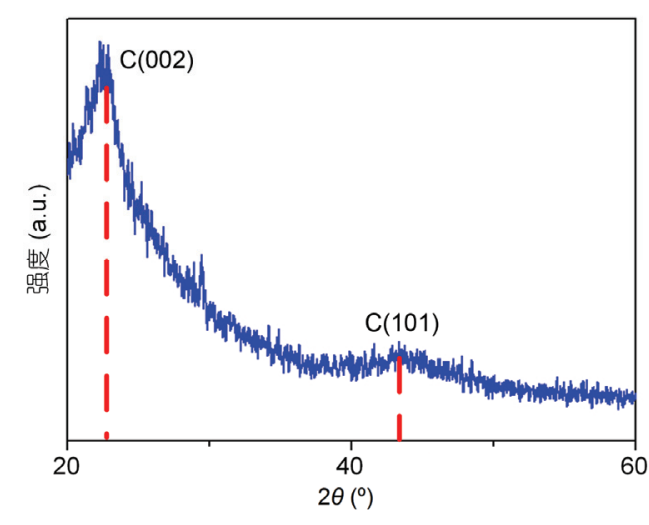

图 5 (网络版彩色) B 批次样品XRD衍射图

Figure 5 (Color online) XRD patterns of sample B

时间分别为 1 和 $2 \mathrm{~h}$ 的碳基电极样品进行了全面的 SEM图像测试, 结果如图6所示. 图6(a)和(d)为2组样 品整体的样貌, 可以看到都是由一些交错复杂的碳 骨架组成, 两者基本形貌一致, 但是 $\mathrm{A}$ 批次样品的碳 骨架有些泛白, 而B批次样品颜色更黑. 由图6(b)和 (e)可以看出, 图6(b)中的孔径比较小; 图6(e)中则可 以看到有很多尺寸不同的孔, 颜色深浅不一, 这是由 一些纳米片形态的碳片组成, 其表面积更大, 会有更 好的电化学性能. 此外, 图6(c)和(f)则可以最为直接 地区分 2 组样品的表征形态, 2 组样品都是由碳骨架组 成, 图6(f)的相邻碳骨架之间没有薄膜层状物质连接; 而图6(c)中可以明显看到薄膜层状物质将碳骨架连
接起来, 并且有一些轻微的断裂, 表明碳化过程没有 完成, 蛋白质变性不完全, 残留了一些薄膜结构. 图 6(c)和(f)的差异进一步验证了 $2 \mathrm{~h}$ 碳化后的样品为多 尺度孔状碳纳米片结构的碳基电极, 比 $1 \mathrm{~h}$ 碳化样品 比表面积更大、碳化程度更深.

\section{3 多孔富氮碳电极的电化学性能分析}

对于双电层电容器的电极材料, 改变电压扫描 方向的瞬间, 电流可以迅速达到稳态, 因而循环伏安 曲线为近矩形对称曲线. 由于 $\mathrm{B}$ 批次电极具有较好的 柔性, 而 $\mathrm{C}$ 批次虽然柔性较差, 但碳化程度较深, 故 对2批次碳化样品进行电化学性能的分析, 可以进一 步探讨不同内应力条件下样品性能的差异. 利用电 化学工作站, 进行了循环伏安法、交流阻抗谱法等性 能测试, 分析 2 组样品的各个电化学参数, 2 批次电化 学性能如图7所示.

首先对 $\mathrm{C}$ 批次碳化样品进行了循环伏安法的测 试, 考虑到三电极系统下水系电解质存在水解问题, 将电位窗口设置为 $-0.5 \sim 0 \mathrm{~V}$, 样品本身重量为 $12 \mathrm{mg}$, 表面积为 $0.5 \mathrm{~cm}^{2}$, 得到的不同电压扫描速率下电流电压图形如图7(a)所示. 结果表明, 随着扫描速率的 增加，图形围成的面积越大，且不同扫描速率下图形 都近似矩形, 说明其为双电层电容工作机理的电极, 稳定性较好. 实验中扫描图形随着循环次数的增加,
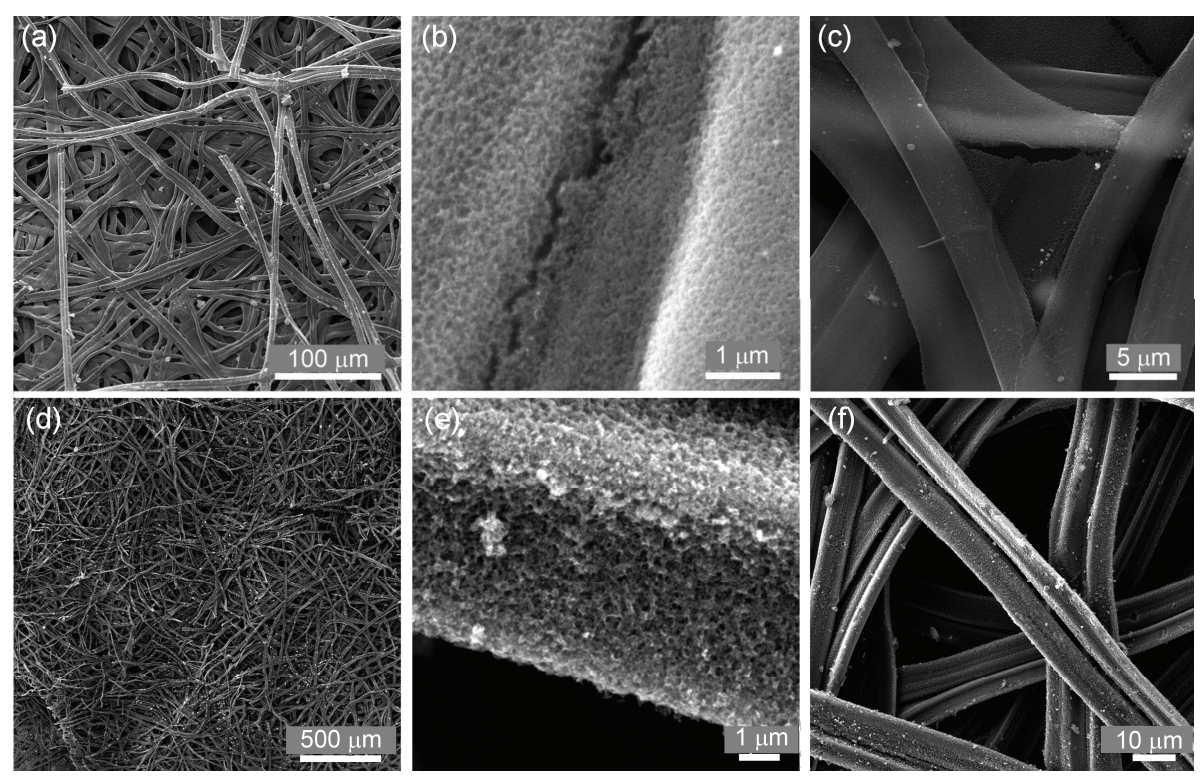

图 6 不同放大尺度下样品表面形貌SEM图像. (a) (c) A批次样品; (d) (f) B批次样品

Figure 6 SEM images of samples in different amplification scale. (a)-(c) Samples A; (d)-(f) sample B 

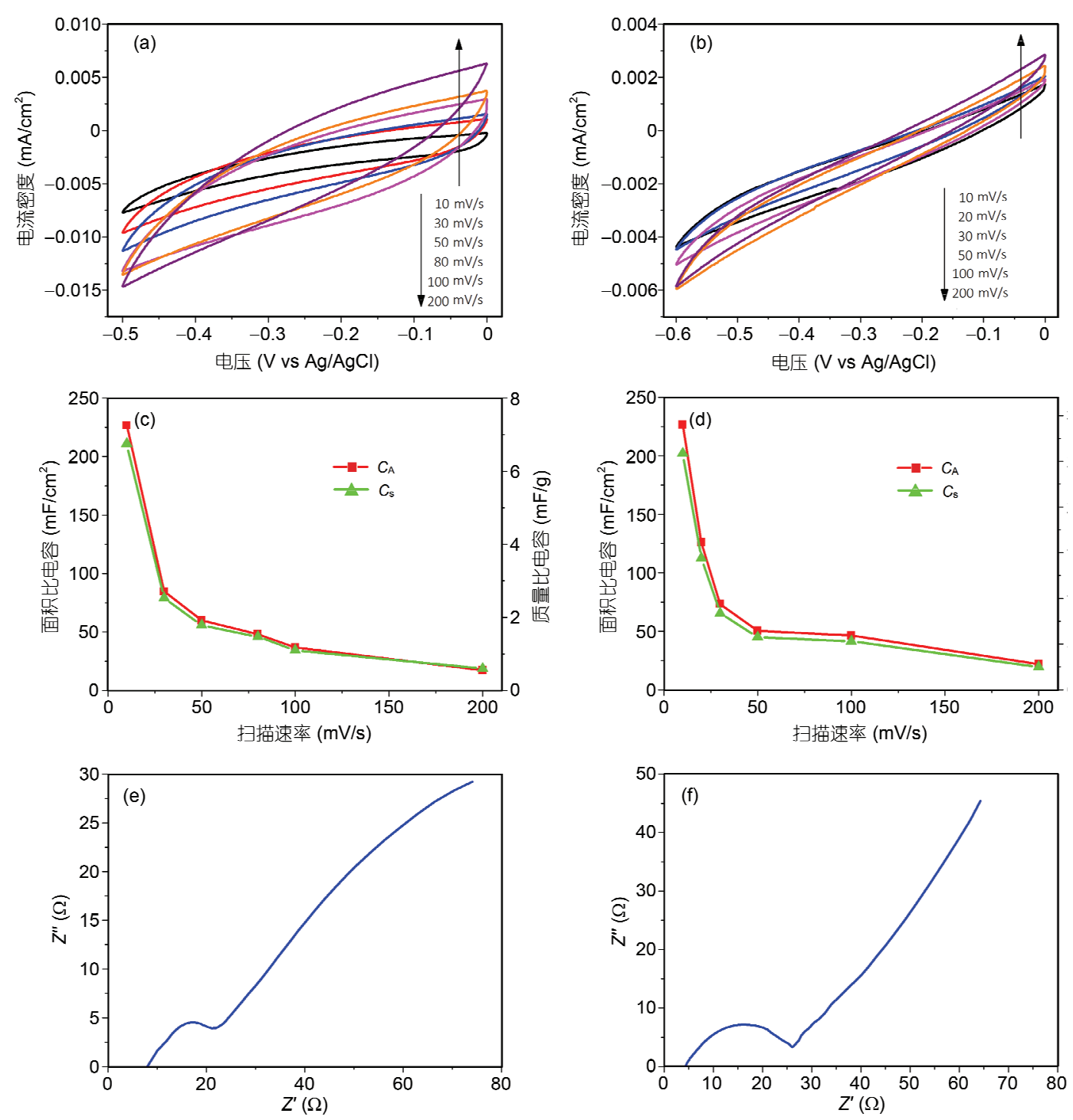

图 7 (网络版彩色)C批次样品(a), (c), (e)和B批次样品(b), (d), (f)的电化学性能. (a), (b) 循环伏安(CV)法; (c), (d) 不同扫描速率下面积比电容与 质量比电容的变化; (e), (f) 交流阻抗谱法

Figure 7 (Color online) Electrochemical performance of samples C (a), (c), (e) and samples B (b), (d), (f) with a three-electrode system at room temperature. (a), (b) CV curves for various scan rates; (c), (d) areal capacitance and specific capacitance as a function of scan rates; (e), (f) Nyquist plots

没有出现衰减, 稳定性很好, 每1次扫描的图形几乎 完全重合.

由循环伏安法计算质量比电容量 $C_{S}$ 与面积比电 容量 $C_{\mathrm{A}}$ :

$$
\begin{aligned}
& C_{\mathrm{S}}=\frac{C}{m}=\frac{1}{k m \Delta V} \int_{V_{1}}^{V_{2}} I(V) \mathrm{d} V, \\
& C_{\mathrm{A}}=\frac{C}{A}=\frac{1}{k A \Delta V} \int_{V_{1}}^{V_{2}} I(V) \mathrm{d} V,
\end{aligned}
$$

式中, $m, A$ 分别为电极上活性物质的质量和面积, $I(V)$ 为 $\mathrm{CV}$ 曲线中的电流响应, 是电压 $V$ 的函数, $V_{1}$ 和 $V_{2}$ 为
电压窗口边界, $k$ 为扫描速率. 据此可以求得不同扫 描速率下的 $C_{\mathrm{S}}$ 和 $C_{\mathrm{A}}($ 图 7(c)), 比电容的值与扫描速 率、电位窗口以及质量或面积有关, 所以扫描速率越 小, 比电容值越大, 与实验结果相符. 当电位扫描速 率为 $10 \mathrm{mV} / \mathrm{s}$ 时, $C_{\mathrm{A}}$ 为 $227 \mathrm{mF} / \mathrm{cm}^{2}, C_{\mathrm{S}}$ 则为 $6.75 \mathrm{~F} / \mathrm{g}$, 性能比普通未处理碳电极有所升高. 由于碳基材料 自身比电容较小, 与其他噟电容电极材料相比, 比电 容值会小一些, 但仍有可以应用的潜力.

此外, 还进行了交流阻抗谱的分析, 设置的频率 为 $0.1 \mathrm{~Hz} 100 \mathrm{kHz}$, 结果如图7(e)所示. 高频下, 阻抗 
较小, 而随着频率的减小, 阻抗成线性上升的趋势, 近乎于平行虚轴, 说明电容特性良好, 其等效串联阻 抗约为 $8 \Omega$, 是比较理想的电极.

接下来对 $\mathrm{B}$ 批次样品做了相同测试, 循环伏安测 试、比电容值以及交流阻抗谱测试结果分别如图7(b), (d)和(f)所示, 使用的碳化样品质量仅为 $7 \mathrm{mg}$, 表面 积为 $0.6 \mathrm{~cm}^{2}$. 图7(b) 可以看出, 电流-电压曲线所选 择的电位窗口为 $-0.6 \sim 0 \mathrm{~V}$, 测试了不同扫描速率下 的电流密度. 随扫描速率的增加, 电流密度变大, 具 有一定的矩形性; 但与 $\mathrm{C}$ 批次相比, 1 个周期所围成面 积偏小, 也与电阻较大有关.

通过计算得到不同扫描速率下的 $C_{\mathrm{A}}$ 和 $C_{\mathrm{S}}$, 结果 表明, 由于其自身质量很小, 因而得到的比电容值更 大(图7(d)). 扫描速率为 $10 \mathrm{mV} / \mathrm{s}$ 时, $C_{\mathrm{A}}$ 可以达到 226 $\mathrm{mF} / \mathrm{cm}^{2}, C_{\mathrm{S}}$ 则为 $26 \mathrm{~F} / \mathrm{g}$, 比普通碳电极有了明显提升, 有作为新型碳基电极的潜质. 而交流阻抗谱分析 (图 7(f))结果可以看出, 在 $0.1 \mathrm{~Hz} 100 \mathrm{kHz}$ 时, 与 C批次 图形相类似, 高频下其等效串联阻抗很小, 阻抗值大 约仅为 $4 \Omega$, 显示了很好的电容特性.

综上所述, 虽然 $\mathrm{B}$ 批次碳化样品在循环伏安测试 中所围成面积略小于 $\mathrm{C}$ 批次, 但是由于自身质量很 轻, 其比电容比 $\mathrm{C}$ 批次更好, 并且 $\mathrm{B}$ 批次的等效串联
阻抗更小, 因而可以同时满足柔性与电化学特性良 好的标准. 该碳基电极制备工艺简单、生物兼容性好, 具有作为柔性固态超级电容器电极的潜质.

\section{3 结论}

随着能量采集纳米发电机和可穿戴电子设备的 深人研究, 如何高效存储收集到的环境中的能量是 下一步亟待解决的问题, 而柔性固态超级电容器所 展现出的优势为上述问题提供了一种很好的解决方 案, 受到了各界的广泛关注. 本文提出了一种简便可 行的制备工艺, 直接对龺茧进行碳化, 得到具有多孔 结构的碳电极. 由于虫茧富含蛋白质, 制得的电极氮 元素比例较高, 对提升电极的电化学性能有较大帮 助, 并结合一系列表征及电化学性能测试, 得出了不 同扫描速率下比电容值的变化趋势和不同频率下碳 化电极阻抗的差异, 最大 $C_{\mathrm{A}}$ 为 $226 \mathrm{mF} / \mathrm{cm}^{2}, C_{\mathrm{S}}$ 则为 $26 \mathrm{~F} / \mathrm{g}$, 等效串联阻抗 $4 \Omega$. 基于此, 进一步优化碳基 电极的工艺与参数, 探讨不同碳化温度与时间对电 极性能的影响, 并以此为基础, 制备出柔性固态超级 电容器, 全面分析其电化学性能, 展示了其在驱动 电子器件和可穿戴设备上的优势, 具有很好的应用 前景.

\section{参考文献}

1 Ayres R U, Turton H, Casten T. Energy efficiency, sustainability and economic growth. Energy, 2007, 32: 634-648

2 Midilli A, Dincer I. Key strategies of hydrogen energy systems for sustainability. Int J Hydrogen Energy, 2007, 32: 511-524

3 Arunachalam V S, Fleischer E L. The global energy landscape and materials innovation. MRS Bull, 2008, 33: 264-288

4 Cao R, Yang Z L. Energy storage technologies in renewable energy electricity generation system. Adv Mater Res, 2012, 462: 225-232

5 Winter M, Brodd R J. What are batteries, fuel cells, and supercapacitors? Chem Rev, 2004, 104: 4245-4270

6 Miller J R, Simon P. Electrochemical capacitors for energy management. Science, 2008, 321: 651-652

7 Pech D, Brunet M, Durou H, et al. Ultrahigh-power micrometre-sized supercapacitors based on onion-like carbon. Nat Nanotechnol, 2010, 5: 651-654

8 Naoi K, Naoi W, Aoyagi S. New generation “nanohybrid supercapacitor”. Acc Chem Res, 2012, 46: 1075-1083

9 Lu X, Yu M, Wang G, et al. Flexible solid-state supercapacitors: Design, fabrication and applications. Energy Environ Sci, 2014, 7: 2160-2181

10 Cheng Y, Zhang H, Lu S, et al. Flexible asymmetric supercapacitors with high energy and high power density in aqueous electrolytes. Nanoscale, 2013, 5: 1067-1073

11 Cheng H, Dong Z, Hu C, et al. Textile electrodes woven by carbon nanotube-graphene hybrid fibers for flexible electrochemical capacitors. Nanoscale, 2013, 5: 3428-3434

12 Simon P, Gogotsi Y. Capacitive energy storage in nanostructured carbon-electrolyte systems. Acc Chem Res, 2012, 46: 1094-1103

$13 \mathrm{Lu} \mathrm{X}$, Zeng Y, Yu M, et al. Oxygen-deficient hematite nanorods as high-performance and novel negative electrodes for flexible asymmetric supercapacitors. Adv Mater, 2014, 26: 3148-3155

14 Meng Y, Zhao Y, Hu C, et al. All-graphene core-sheath microfibers for all-solid-state, stretchable fibriform supercapacitors and wearable electronic Textiles. Adv Mater, 2013, 25: 2326-2331 
15 Jost K, Stenger D, Perez C R. Knitted and screen printed carbon-fiber supercapacitors for applications in wearable electronics. Energy Environ Sci, 2013, 6: 2698-2705

16 Weng Z, Su Y, Wang D W, et al. Graphene-cellulose paper flexible supercapacitors. Adv Energy Mater, 2011, 1: 917-922

17 Zang X, Chen Q, Li P, et al. Highly flexible and adaptable, all-solid-state supercapacitors based on graphene woven-fabric film electrodes. Small, 2014, 10: 2583-2588

18 Sivakkumar S R, Kim W J, Choi J A. Electrochemical performance of polyaniline nanofibres and polyaniline/multi-walled carbon nanotube composite as an electrode material for aqueous redox supercapacitors. J Power Sources, 2007, 171: 1062-1068

19 Niu Z, Dong H, Zhu B, et al. Highly stretchable, integrated supercapacitors based on single-walled carbon nanotube films with continuous reticulate architecture. Adv Mater, 2013, 25: 1058-1064

20 Jiang S, Shi T, Zhan X, et al. High-performance all-solid-state flexible supercapacitors based on two-step activated carbon cloth. J Power Sources, 2014, 272: 16-23

21 Tai Z, Yan X, Lang J W, et al. Enhancement of capacitance performance of flexible carbon nanofiber paper by adding graphene nanosheets. J Power Sources, 2012, 199: 373-378

22 Wang Y G, Wang Z D, Xia Y Y. An asymmetric supercapacitor using $\mathrm{RuO}_{2} / \mathrm{TiO}_{2}$ nanotube composite and activated carbon electrodes. Electrochim Acta, 2005, 50: 5641-5646

23 Qie L, Chen W M, Wang Z H, et al. Nitrogen-doped porous carbon nanofiber webs as anodes for lithium ion batteries with a superhigh capacity and rate capability. Adv Mater, 2012, 24: 2047-2050

24 Qie L, Chen W, Xu H, et al. Synthesis of functionalized 3D hierarchical porous carbon for high-performance supercapacitors. Energy Environ Sci, 2013, 6: 2497-2504

25 Yun Y S, Cho S Y, Shim J, et al. Microporous carbon nanoplates from regenerated silk proteins for supercapacitors. Adv Mater, 2013, 25: 1993-1998

26 Li Y, Li Z, Shen P K. Simultaneous formation of ultrahigh surface area and three-dimensional hierarchical porous graphene-like networks for fast and highly stable supercapacitors. Adv Mater, 2013, 17: 2474-2480

27 Liang Y, Wu D, Fu R. Carbon microfibers with hierarchical porous structure from electrospun fiber-like natural biopolymer. Sci Rep, 2013, 3: 1119-1123

28 Li Z, Xu Z, Tan X, et al. Mesoporous nitrogen-rich carbons derived from protein for ultra-high capacity battery anodes and supercapacitors. Energy Environ Sci, 2013, 6: 871-878

29 Dutta S, Kevin A B, Wu C W. Hierarchically porous carbon derived from polymers and biomass: Effect of interconnected pores on energy applications. Energy Environ Sci, 2014, 7: 3574-3592

30 Hou J H, Cao C B, Idrees F, et al. Hierarchical porous nitrogen-doped carbon nanosheets derived from silk for ultrahigh-capacity battery anodes and supercapacitors. ACS Nano, 2015, 9: 2526-2564 


\title{
Fabrication and characterization analysis of flexible porous nitrogen-doped carbon-based supercapacitor electrodes
}

\author{
SONG Yu, MENG Bo, CHEN XueXian, CHEN HaoTian, HAN MengDi, \\ CHENG XiaoLiang \& ZHANG HaiXia \\ National Key Lab of Nano/Micro Fabrication Technology, Institute of Microelectronics, Peking University, Beijing 100871, China
}

Supercapacitor as a new-type energy storage device has attracted increasing interests, because it can provide substantially higher specific/volumetric energy density compared to conventional capacitors, and higher power density and longer cycling lifetime compared to Li-ion batteries. Therefore, it shows great promise as green energy storage device for back-up energy systems and electronic vehicles. Among all kinds of supercapacitors, flexible solid-state supercapacitor, which consists of flexible electrodes, solid-state electrolyte and separator, has attracted much attention due to its potential abilities in wearable electronics and flexible devices. Numerous efforts have been devoted to fabricate flexible electrodes because the fundamental limit of energy storage capability is largely determined by the electrode material. Therefore, how to fabricate suitable flexible electrodes alerts significant influence on supercapacitors' performance. Compared to pseudocapacitive materials, carbon-based materials have been widely investigated for flexible electrodes due to their good electrical conductivity, excellent cycling stability, high power density and outstanding mechanical properties. In this work, an effective and feasible method to fabricate carbon-based electrode for the fabrication of flexible and high-performance supercapacitors has been proposed. In order to find the relatively suitable electrode, samples under different experimental conditions have been tested and analyzed systematically. Through the simultaneous activation and carbonization process of the biomass-derived natural silk, all of these carbon-based electrodes have a few features, like hierarchical porous, rich $\mathrm{N}$-doped and carbon nanosheet structure. The as-obtained electrodes show favorable capabilities for electrochemical energy storage. The specific/mass capacitor of the best carbon-based electrode can achieve 226 $\mathrm{mF} / \mathrm{cm}^{2}$ and $26 \mathrm{~F} / \mathrm{g}$, respectively, and the equivalent series resistance of it is only $4 \Omega$, thus showing satisfactory electrochemical performance. In conclusion, the excellent capacitance performance of the ultralight and flexible nanomaterials makes them promising candidates as electrode materials. Attributed to the good flexibility and ultralight weight, this proposed electrodes can be directly fabricated into flexible solid-state supercapacitor without any binder and conductive agents.

flexible electrode, supercapacitor, carbonization, electrochemical specific capacitance

doi: 10.1360/N972015-01080 